\title{
ISOPERIMETRIC INEQUALITY UNDER KÄHLER RICCI FLOW
}

\author{
GANG TIAN AND QI S. ZHANG
}

\begin{abstract}
Let $(\mathbf{M}, g(t))$ be a Kähler Ricci flow with positive first Chern class. First, we prove a uniform isoperimetric inequality for all time. In the process, we also prove a Cheng-Yau type log gradient bound for positive harmonic functions on $(\mathbf{M}, g(t))$ and a Poincaré inequality without assuming the Ricci curvature is bounded from below.
\end{abstract}

\section{INTRODUCTION}

In this paper we study Kähler Ricci flows

$$
\partial_{t} g_{i \bar{j}}=-R_{i \bar{j}}+g_{i \bar{j}}=\partial_{i} \partial_{\bar{j}} u, \quad t>0
$$

on a compact, Kähler manifold $\mathbf{M}$ of complex dimension $m=n / 2$, with positive first Chern class.

Given initial Kähler metric $g_{i \bar{j}}(0)$, H. D. Cao [Ca proved that (1.1) has a solution for all time $t$. Recently, many results concerning long time and uniform behavior of (1.1) have appeared. For example, when the curvature operator or the bisectional curvature is nonnegative, it is known that solutions to (1.1) stays smooth when time goes to infinity (see [CCZ], CT1] and [CT2 for examples). In the general case, Perelman (cf [ST]) proved that the scalar curvature $R$ is uniformly bounded, and the Ricci potential $u(\cdot, t)$ is uniformly bounded in $C^{1}$ norm, with respect to $g(t)$. When the complex dimension $m=2$, let $(\mathbf{M}, g(t))$ be a solution to (1.1), it is proved in ([CW]) that the isoperimetric constant for $(\mathbf{M}, g(t))$ is bounded from below by a uniform constant. We mention that an isoperimetric estimate for the Ricci flow on the two sphere was already proven by Hamilton in [Ha].

In this paper, we prove that in all complex dimensions, the isoperimetric constant for $(\mathbf{M}, g(t))$ is bounded from below by a uniform constant. This extends the result of ChenWang mentioned above. This result seem to add more weight to the belief that the Kähler Ricci flow converges to a Kähler Ricci soliton as $t \rightarrow \infty$, except on a subvariety of complex codimension 2 ,

To make the statement precise, let's introduce notations and definition. We use $\mathbf{M}$ to denote a compact Riemann manifold and $g(t)$ to denote the metric at time $t ; d(x, y, t)$ is the geodesic distance under $g(t) ; B(x, r, t)=\{y \in \mathbf{M} \mid d(x, y, t)<r\}$ is the geodesic ball of radius $r$, under metric $g(t)$, centered at $x$, and $|B(x, r, t)|_{g(t)}$ is the volume of $B(x, r, t)$ under $g(t) ; d g(t)$ is the volume element. We also reserve $R=R(x, t)$ as the scalar curvature under $g(t)$. When the time variable $t$ is not explicitly used, we may also suppress it in the notations mentioned above.

The main result of the paper is the following theorem.

Theorem 1.1. Let $(\mathbf{M}, g(t)), \partial_{t} g_{i \bar{j}}=-R_{i \bar{j}}+g_{i \bar{j}}$, be a Kähler Ricci flow on a $n$ real dimensional compact, Kähler manifold with positive first Chern class. Then there exists a 
uniform constant $S_{0}$, depending only on the initial metric $g(0)$ and a numerical constant $C$, such that

$$
\left[\int_{\mathbf{M}}|u|^{n /(n-1)} d g(t)\right]^{(n-1) / n} \leq S_{0} \int_{\mathbf{M}}|\nabla u| d g(t)+\frac{C}{|\mathbf{M}|_{g(t)}^{1 / n}} \int_{\mathbf{M}}|u| d g(t)
$$

for all $u \in C^{\infty}(\mathbf{M})$.

Remark.

It is well known that Theorem 1.1 implies a uniform lower bound for the isoperimetric constant of $(\mathbf{M}, g(t))$, i.e. there exists a positive constant $c_{0}$, depending only on the initial metric such that

$$
I(\mathbf{M}, g(t)) \equiv \inf _{D \subset \mathbf{M}} \frac{|\partial D|}{[\min \{|D|,|\mathbf{M}-D|\}]^{(n-1) / n}} \geq c_{0} .
$$

Here all the volume are with respect to $g(t)$; and $D$ is a subdomain of $\mathbf{M}$ such that $\partial D$ is a $n-1$ dimensional submanifold of $\mathbf{M}$. A proof can be found in [CLN] Section 5.1 e.g.

The proof of the theorem is based on the following properties for Kähler Ricci flow on a compact manifold with positive first Chern class.

Property A. Let $(\mathbf{M}, g(t))$ be a Kähler Ricci flow (1.1) on a compact manifold with positive first Chern class. There exist uniform positive constants $C$, and $\kappa$ so that

1. $|R(g(t))| \leq C$

2. $\operatorname{diam}(\mathbf{M}, g(t)) \leq C$,

3. $\|u\|_{C^{1}} \leq C$.

4. $|B(x, r, t)|_{g(t)} \geq \kappa r^{n}$, for all $t>0$ and $r \in(0, \operatorname{diam}(\mathbf{M}, g(t)))$.

5. $|B(x, r, t)|_{g(t)} \leq \kappa^{-1} r^{n}$ for all $r>0, t>0$.

Property B. Under the same assumption as in Property A, there exists a uniform constant $S_{2}$ so that the following $L^{2}$ Sobolev inequality holds:

$$
\left(\int_{\mathbf{M}} v^{2 n /(n-2)} d g(t)\right)^{(n-2) / n} \leq S_{2}\left(\int_{\mathbf{M}}|\nabla v|^{2} d g(t)+\int_{\mathbf{M}} v^{2} d g(t)\right)
$$

for all $v \in C^{\infty}(\mathbf{M}, g(t))$.

Property A 1-4 is due to Perelman (c.f. [ST]), Property B was first established in [Z07] (see also [Ye], [Z10] ). Property A 5 can be found in [Z11] and also [CW2].

The rest of the paper is organized as follows. In Section 2, we prove some gradient bounds for harmonic functions on $(\mathbf{M}, g(t))$. Since the bounds do not rely on the usual lower bound of Ricci curvature, the result may be of independent interest. Using these bounds, we prove the theorem in Section 3. 


\section{GRADIENT BOUNDS FOR HARMONIC FUNCTIONS}

In order to prove the theorem, in this section we state and prove a number of results on harmonic functions on certain manifolds with fixed metric. These results are well known when the manifold has nonnegative Ricci curvature, a property that is not available for us. Since some of these results may be of independent interest, we will also deal with the real variable case and impose some conditions which are more general than needed for the proof of the theorems in Section 1. As the metric is independent of time in this section, we will suppress the time variable $t$.

In this section, the basic assumptions on the $n$ real dimensional manifolds $\mathbf{M}$ are

Assumption 1. $L^{2}$ Sobolev inequality: there is a positive constant $\alpha$ such that

$$
\left(\int_{\mathbf{M}} u^{2 n /(n-2)} d g(t)\right)^{(n-2) / n} \leq \alpha\left(\int_{\mathbf{M}}|\nabla u|^{2} d g(t)+\int_{\mathbf{M}} u^{2} d g(t)\right)
$$

for all $u \in C^{\infty}(\mathbf{M})$.

Assumption 2. There exists a positive constant $\kappa$, such that

$$
\kappa r^{n} \leq|B(x, r)| \leq \kappa^{-1} r^{n}, \quad x \in \mathbf{M}, \quad 0<r<\operatorname{diam}(\mathbf{M}) \leq 1 .
$$

Assumption 3. There exists a smooth function $L=L(x)$ and 2 smooth parallel $(2,2)$ tensor fields $P$ and $Q$ such that the Ricci curvature is given by

$$
R_{i j}=P_{i j}^{k l} \partial_{k} \partial_{l} L+Q_{i j}^{k l} g_{k l}
$$

under a local coordinates. Moreover $\|P\|_{\infty} \leq 1,\|Q\|_{\infty} \leq 1$. Here $\partial_{k} \partial_{l} L$ is the Hessian of $L$.

Note that Assumption 3 includes as a special case, the formula for the Ricci curvature on Kähler manifolds $\partial_{i} \partial_{\bar{j}} u=g_{i \bar{j}}-R_{i \bar{j}}$ where $u$ is the Ricci potential.

Lemma 2.1. Suppose $(\mathbf{M}, g)$ is a compact Riemann manifold of real dimension $n$, satisfying Assumptions 1, 2, 3.

Let $u$ be a smooth harmonic function in $B\left(x_{0}, r\right)$ where $x_{0} \in \mathbf{M}$ and $r \leq \operatorname{diam}(\mathbf{M})$. Then there exists a positive constant $C_{0}=C_{0}\left(\alpha, \kappa,\|\nabla L\|_{\infty}\right)$ such that

$$
\sup _{x \in B\left(x_{0}, r / 2\right)}|\nabla u(x)| \leq C_{0} \frac{1}{r}\left(\frac{1}{r^{n}} \int_{B\left(x_{0}, r\right)} u^{2} d g\right)^{1 / 2} .
$$

Proof. Since $u$ solves $\Delta u=0$, by Bochner's formula, we have

$$
\Delta|\nabla u|^{2}=2|H e s s u|^{2}+2 \operatorname{Ric}(\nabla u, \nabla u) .
$$

Given $\sigma \in(0,1)$, let $\psi=\psi(x)$ be a standard Lipschitz cut-off function such that $\psi(x)=0$ when $x \in B\left(x_{0}, r\right)^{c} ; 0 \leq \psi \leq 1$ and $\psi(x)=1$ when $x \in B\left(x_{0}, \sigma r\right)$ and $|\nabla \psi| \leq \frac{4}{(1-\sigma) r}$. We mention that no second order derivatives of $\psi$ are involved. So we only need $\psi$ is Lipschitz.

For clarity of presentation, we write

$$
f=|\nabla u|^{2} .
$$


Given a number $p \geq 1$, using $f \psi^{2}$ as a test function on (2.1), after a routine calculation, we derive

$$
\begin{aligned}
& \frac{2 p-1}{p^{2}} \int_{B\left(x_{0}, r\right)}\left|\nabla\left(f^{p} \psi\right)\right|^{2} d g \\
& \leq \frac{C}{(1-\sigma)^{2} r^{2}} \int_{B\left(x_{0}, r\right)} f^{2} d g-2 \int_{B\left(x_{0}, r\right)}|H e s s u|^{2} f^{2 p-1} d g-2 \int_{B\left(x_{0}, r\right)} \operatorname{Ric}(\nabla u, \nabla u) f^{2 p-1} \psi^{2} d g \\
& \equiv I_{1}+I_{2}+I_{3} .
\end{aligned}
$$

Now we want to absorb part of $I_{3}$ by $I_{2}$ which is a good term. In a local orthonormal coordinates, we denote $u_{i}$ the i-th component of $\nabla u$. Then By Assumption 3, we have, after integrating by parts,

$$
\begin{aligned}
I_{3}= & -2 \int R_{i j} u_{i} u_{j} f^{2 p-1} \psi^{2} d g \\
= & -2 \int P_{i j}^{k l}\left(\partial_{k} \partial_{l} L\right) u_{i} u_{j} f^{2 p-1} \psi^{2} d g-2 \int Q_{i j}^{k l} g_{k l} u_{i} u_{j} f^{2 p-1} \psi^{2} d g \\
= & 2 \int P_{i j}^{k l}\left(\partial_{l} L\right)\left(\partial_{k} u_{i}\right) u_{j} f^{2 p-1} \psi^{2} d g+2 \int P_{i j}^{k l}\left(\partial_{l} L\right) u_{i}\left(\partial_{k} u_{j}\right) f^{2 p-1} \psi^{2} d g \\
& \quad+2 \int P_{i j}^{k l}\left(\partial_{l} L\right) u_{i} u_{j} \partial_{k}\left(f^{2 p-1} \psi^{2}\right) d g-2 \int Q_{i j}^{k l} g_{k l} u_{i} u_{j} f^{2 p-1} \psi^{2} d g
\end{aligned}
$$

Here we also used the assumption that the $P$ tensor is parallel. To control the second from last term in the above identity, we notice that $\left|u_{i} u_{j}\right| \leq|\nabla u|^{2}=f$ and that

$$
f \partial_{k}\left(f^{2 p-1} \psi^{2}\right)=\left(\partial_{k} f^{p}\right) \psi^{2} \frac{2 p-1}{p} f^{p}+2 f^{2 p}\left(\partial_{k} \psi\right) \psi
$$

From here, using Young's inequality, it is easy to see that

$I_{3} \leq \frac{1}{2} \int|\nabla(f \psi)|^{2} d g+\int|H e s s u|^{2} f^{2 p-1} \psi^{2} d g+C\left(\frac{\|\nabla L\|_{\infty}^{2}}{[(1-\sigma) r]^{2}}+\|\nabla L\|_{\infty}^{2}+1\right) \int f^{2 p} \psi^{2} d g$.

Substituting this to (2.2), we deduce

$$
\int\left|\nabla\left(f^{p} \psi\right)\right|^{2} d g \leq C p\left(\frac{\|\nabla L\|_{\infty}^{2}}{[(1-\sigma) r]^{2}}+\|\nabla L\|_{\infty}^{2}+1\right) \int f^{2 p} \psi^{2} d g
$$

Since $\operatorname{diam}(\mathbf{M}) \leq 1$ by Assumption 2, the last inequality implies

$$
\int\left|\nabla\left(f^{p} \psi\right)\right|^{2} d g \leq C p \frac{\|\nabla L\|_{\infty}^{2}+1}{[(1-\sigma) r]^{2}} \int f^{2 p} \psi^{2} d g
$$

Using the $L^{2}$ Sobolev inequality in Assumption 1 and Moser's iteration, we deduce, by choosing $p=(n /(n-2))^{i}$, and replacing $(1-\sigma) r$ by $(1 / 4)^{i} r, i=0,1,2, \ldots$, that

$$
\sup _{x \in B\left(x_{0}, r / 2\right)}|\nabla u(x)|^{2} \leq C_{1} \frac{1}{r^{n}} \int_{B\left(x_{0}, 3 r / 4\right)}|\nabla u|^{2} d g
$$

where $C_{1}=C_{1}\left(\alpha, \kappa,\|\nabla L\|_{\infty}\right)$. We observe that even though that the number $p$ appears on the right hand side of the inequality before (2.3), but its growth is only an exponential of $i$. As well known, it will be suppressed by the Moser iteration process, just like the term $1 /(1-\sigma)^{2}$. 
Next we take $\sigma=3 / 4$ in the definition of the cut off function $\psi$. Using $u \psi^{2}$ as a test function on $\Delta u=0$, we infer, after a routine calculation

$$
\int_{B\left(x_{0}, 3 r / 4\right)}|\nabla u|^{2} d g \leq \frac{C}{r^{2}} \int_{B\left(x_{0}, r\right)} u^{2} d g .
$$

Here $C$ is a numerical constant. Combining the last two inequalities we arrive at

$$
\sup _{x \in B\left(x_{0}, r / 2\right)}|\nabla u(x)|^{2} \leq C_{0} \frac{1}{r^{n+2}} \int_{B\left(x_{0}, r\right)} u^{2} d g
$$

where $C_{0}=C_{0}\left(\alpha, \kappa,\|\nabla L\|_{\infty}\right)$.

The next lemma is simply the $L^{2}$ mean value inequality for the Laplace and heat equation under Assumptions 1 and 2. Since the result is well known (Grigoryan Gr] and Saloff-Coste [Sa]), we omit the proof.

Lemma 2.2. Let $\mathbf{M}$ be a manifold satisfying Assumptions 1, 2.

Suppose $u$ be is a smooth harmonic function in $B\left(x_{0}, r\right)$ where $x_{0} \in \mathbf{M}$ and $r \leq$ $\operatorname{diam}(\mathbf{M})$. Then there exists a positive constant $C_{1}=C_{1}(\alpha, \kappa)$ such that

$$
\sup _{x \in B\left(x_{0}, r / 2\right)}|u(x)| \leq C_{1}\left(\frac{1}{r^{n}} \int_{B\left(x_{0}, r\right)} u^{2} d g\right)^{1 / 2} .
$$

Suppose $u$ is a solution of the heat equation $\Delta u-\partial_{t} u=0$ in the space time cube $B\left(x_{0}, r\right) \times\left[t_{0}-r^{2}, t_{0}\right]$. Then

$$
\sup _{(x, t) \in B\left(x_{0}, r / 2\right) \times\left[t_{0}-r^{2} / 4\right]}|u(x, t)| \leq C_{1}\left(\frac{1}{r^{n+2}} \int_{t-r^{2}}^{t} \int_{B\left(x_{0}, r\right)} u^{2} d g d s\right)^{1 / 2} .
$$

The next lemma provides bounds for the Green's function of the Laplacian and its gradients.

Lemma 2.3. Let $\mathbf{M}$ be a manifold satisfying Assumptions 1, 2 and 3. Assume also $\operatorname{diam}(\mathbf{M})>\beta>0$ for a positive constant $\beta$. Let $\Gamma_{0}$ be the Green's function of the Laplacian $\Delta$ on $\mathbf{M}$. Then there exists a positive constant $C_{0}=\left(\alpha, \beta, \kappa,\|\nabla L\|_{\infty}\right)$ such that

(a). $\left|\Gamma_{0}(x, y)\right| \leq \frac{C_{0}}{d(x, y)^{n-2}}, x, y \in \mathbf{M}$,

(b). $\left|\nabla_{x} \Gamma_{0}(x, y)\right| \leq \frac{C_{0}}{d(x, y)^{n-1}}, x, y \in \mathbf{M}$.

Proof. Once (a) is proven, (b) is just a consequence of (a) and Lemma 2.1 applied on the ball $B(x, d(x, y) / 2)$. So now we just need to prove (a).

On a compact manifold $\mathbf{M}$, we know that

$$
\Gamma_{0}(x, y)=\int_{0}^{\infty}\left(G(x, t, y)-\frac{1}{|\mathbf{M}|}\right) d t
$$

where $G$ is the fundamental solution of the heat equation $\Delta u-\partial_{t} u=0$. We remark that the metric is fixed here. So we need to bound $G$. Under Assumptions 1 and 2, Grigoryan [Gr] and Saloff-Coste [Sa] proved that there exist positive constants $A_{1}, A_{2}, A_{3}$ which depend only on $\alpha$ and $\kappa$ such that

$$
G(x, t, y) \leq A_{1}\left(1+\frac{1}{t^{n / 2}}\right) e^{-A_{2} d(x, y)^{2} / t} .
$$


Fixing $x, y$ and $t$, we write $u=u(z, l)=G(z, l, y)$ and regard it as a solution of the heat equation in the cube $B(x, r) \times\left[t-r^{2}, t\right]$. Here $r=\sqrt{t} / 2$. Extending Lemma 2.1 to the parabolic case in a routine manner, we know that

$$
|\nabla u(x, t)| \leq \frac{C_{1}}{r}\left(\frac{1}{r^{n+2}} \int_{t-r^{2}}^{t} \int_{B(x, r)} u^{2} d g d s\right)^{1 / 2} .
$$

Substituting (2.5) to the right hand side, we know that

$$
\left|\nabla_{x} G(x, t, y)\right| \leq A_{1}\left(1+\frac{1}{t^{(n+1) / 2}}\right) e^{-A_{2} d(x, y)^{2} / t} .
$$

Here the constants $A_{1}$ and $A_{2}$ may have changed. It is well known that this gradient bound and the upper bound (2.5) together imply a Gaussian lower bound for the heat kernel $G$. See $[\mathrm{CD}]$, p1165 e.g. Now, by [Sa], the following $L^{2}$ Poincaré inequality holds: for any $u \in C^{\infty}(\mathbf{M}), r \in(0, \operatorname{diam}(\mathbf{M})]$,

$$
\int_{B(x, r / 2)}\left|u-\bar{u}_{B(x, r / 2)}\right|^{2} d g \leq A_{3} r^{2} \int_{B(x, r)}|\nabla u|^{2} d g .
$$

By a trick in Jerison [J], which uses only volume doubling property, one has that

$$
\int_{B(x, r)}\left|u-\bar{u}_{B(x, r)}\right|^{2} d g \leq C A_{3} r^{2} \int_{B(x, r)}|\nabla u|^{2} d g
$$

Here $C$ depends only on $\kappa$. We mention that some of the cited results were stated for complete noncompact manifolds. But they are also valid for complete, closed manifolds as long as the diameters are uniformly bounded.

Let $u_{0} \in C^{\infty}(\mathbf{M})$ be a function such that $\int_{\mathbf{M}} u_{0} d g=0$. Then the function

$$
u(x, t)=\int_{\mathbf{M}}\left(G(x, t, z)-\frac{1}{|\mathbf{M}|}\right) u_{0}(z) d g(z)
$$

is a solution to the heat equation such that $\int_{\mathbf{M}} u(x, t) d g(x)=0$. By the $L^{2}$ Poincaré inequality with $r=\operatorname{diam}(\mathbf{M})$, we have

$$
\int_{\mathbf{M}} u^{2} d g \leq C A_{3} \operatorname{diam}(\mathbf{M})^{2} \int_{\mathbf{M}}|\nabla u|^{2} d g \leq C A_{3} \int_{\mathbf{M}}|\nabla u|^{2} d g
$$

since $\operatorname{diam}(\mathbf{M}) \leq 1$ by assumption. From this we deduce

$$
\frac{d}{d t} \int_{\mathbf{M}} u^{2} d g=-2 \int_{\mathbf{M}}|\nabla u|^{2} d g=-2\left(C A_{3}\right)^{-1} \int_{\mathbf{M}} u^{2} d g
$$

and consequently

$$
\int_{\mathbf{M}} u^{2}(z, s) d g \leq e^{-2\left(C A_{3}\right)^{-1} s} \int_{\mathbf{M}} u_{0}^{2}(z) d g, \quad s>0
$$

Recall that we assume $\operatorname{diam}(\mathbf{M})>\beta>0$. For $t \geq \beta^{2}$, we can apply Lemma 2.2 to get

$$
u^{2}(x, t) \leq C_{1}^{2} \frac{1}{\beta^{n+2}} \int_{t-\beta^{2}}^{t} \int_{\mathbf{M}} u^{2}(z, s) d g d s .
$$

Combining this with the previous inequality, we arrive at

$$
u^{2}(x, t) \leq C_{2} e^{-2\left(C A_{3}\right)^{-1} t} \int_{\mathbf{M}} u_{0}^{2}(z) d g
$$


where $C_{2}=C_{0}\left(\alpha, \beta, \kappa, A_{3}\right)$. By (2.8), this means

$$
\left[\int_{\mathbf{M}}\left(G(x, t, z)-\frac{1}{|\mathbf{M}|}\right) u_{0}(z) d g\right]^{2} \leq C_{2} e^{-2\left(C A_{3}\right)^{-1} t} \int_{\mathbf{M}} u_{0}^{2}(z) d g
$$

Fixing $x \in \mathbf{M}$ and $t \geq \beta^{2}$, and taking $u_{0}(z)=G(x, t, z)-\frac{1}{|\mathbf{M}|}$ in the above inequality, we obtain

$$
\int_{\mathbf{M}}\left(G(x, t, z)-\frac{1}{|\mathbf{M}|}\right)^{2} d g \leq C_{2} e^{-2\left(C A_{3}\right)^{-1} t}, \quad t \geq \beta^{2} .
$$

Fixing $x$, the function $h(z, t) \equiv G(x, t, z)-\frac{1}{|\mathbf{M}|}$ is also a solution to the heat equation. Applying the mean value inequality in Lemma 2.2 on the cube $B(y, \beta) \times\left[t-\beta^{2}, t\right]$, we infer

That is

$$
h^{2}(y, t) \leq C_{1}^{2} \frac{1}{\beta^{n+2}} \int_{t-\beta^{2}}^{t} \int_{\mathbf{M}} h^{2}(z, s) d g d s .
$$

$$
\left(G(x, t, y)-\frac{1}{|\mathbf{M}|}\right)^{2} \leq C_{1}^{2} \frac{1}{\beta^{n+2}} \int_{t-\beta^{2}}^{t} \int_{\mathbf{M}}\left(G(x, s, z)-\frac{1}{|\mathbf{M}|}\right)^{2} d g d s .
$$

Substituting (2.9) to the last inequality, we deduce

$$
\left|G(x, t, y)-\frac{1}{|\mathbf{M}|}\right| \leq C_{3} e^{-C_{4} t}, \quad t \geq \beta^{2},
$$

where $C_{3}, C_{4}$ depend only on $\alpha, \beta, \kappa$ and $A_{3}$ which only depends on $\alpha, \kappa$.

From (2.4),

$$
\begin{aligned}
\Gamma_{0}(x, y) & =\int_{0}^{\beta^{2}}\left(G(x, t, y)-\frac{1}{|\mathbf{M}|}\right) d t+\int_{\beta^{2}}^{\infty}\left(G(x, t, y)-\frac{1}{|\mathbf{M}|}\right) d t \\
& \equiv I_{1}+I_{2} .
\end{aligned}
$$

Using the bound (2.5) on $I_{1}$ and (2.10) on $I_{2}$, we derive, after simple integration,

$$
\left|\Gamma_{0}(x, y)\right| \leq \frac{C_{0}}{d(x, y)^{n-2}},
$$

where $C_{0}$ depends only on $\alpha, \beta, \kappa$. This proves part (a) of the Lemma.

As mentioned earlier, part (b) follows from part (a) and Lemma 2.1.

The next result is a Cheng-Yau type log gradient estimate. Although not used in the proof of the theorems, it may be of independent interest.

Proposition 2.1. Let $\mathbf{M}$ be a manifold satisfying Assumptions 1, 2 and 3. Let $u$ be a positive harmonic function in the geodesic ball $B(x, 2 r)$, which is properly contained in M. Then there exists a positive constant $C$, depending only on the controlling constants in Assumptions 1-3, such that

$$
\sup _{B(x, r)}|\nabla \ln u| \leq \frac{C}{r}
$$

when $r \in(0,1]$.

Proof. 
For convenience, we use the following notations

$$
h \equiv \ln u, \quad F \equiv|\nabla h|^{2} .
$$

Following [CY], it is well known that $\Delta h=-F$ and

$$
\Delta F=-2 \nabla h \nabla F+2|H e s s h|^{2}+2 R i c(\nabla h, \nabla h) .
$$

Consider the function

$$
w \equiv F^{5 n}
$$

By a routine calculation, we know that, for any $p \geq 1$,

$$
\Delta w^{p} \geq-2 \nabla h \nabla w^{p}+10 n p F^{5 n p-1}|H e s s h|^{2}+10 n p F^{5 n p-1} \operatorname{Ric}(\nabla h, \nabla h)
$$

Given $\sigma \in(0,1)$, let $\psi=\psi(x)$ be a standard smooth cut-off function such that $\psi(x)=0$ when $x \in B\left(x_{0}, r\right)^{c} ; 0 \leq \psi \leq 1$ and $\psi(x)=1$ when $x \in B\left(x_{0}, \sigma r\right)$ and $|\nabla \psi| \leq \frac{4}{(1-\sigma) r}$. Using $w^{2} \psi$ as a test function on (2.12), we deduce, after a straight forward calculation, that

$$
\begin{aligned}
\int\left|\nabla\left(w^{p} \psi\right)\right|^{2} d g \leq & -10 n p \int F^{5 n p-1} \mid \text { Hess }\left.h\right|^{2} w^{p} \psi^{2} d g+2 \int \nabla h \nabla w^{p} w^{p} \psi^{2} d g \\
& \quad-10 n p \int F^{5 n p-1} \operatorname{Ric}(\nabla h, \nabla h) w^{p} \psi^{2} d g \\
\equiv & I_{1}+I_{2}+I_{3} .
\end{aligned}
$$

Next we will show that the negative term $I_{1}$ dominate $I_{2}$ and $I_{3}$, modulo some harmless terms. Observe that

$$
\begin{aligned}
I_{2} & =\int \psi^{2} \nabla h \nabla w^{2 p} d g \\
& =-2 \int \psi \nabla \psi \nabla h w^{2 p} d g-\int \psi^{2} \Delta h w^{2 p} d g .
\end{aligned}
$$

Recall that $\Delta h=-|\nabla h|^{2}=F$. Hence, by Young's inequality, for any given $\epsilon>0$,

$$
I_{2} \leq(\epsilon+1) \int F w^{2 p} \psi^{2} d g+\epsilon^{-1}\|\nabla \psi\|_{\infty}^{2} \int w^{2 p} \psi^{2} d g
$$

It takes a little longer to prove the bound for $I_{3}$. By our condition on the Ricci curvature $R_{i j}$, we have

$$
I_{3}=-10 n p \int F^{5 n p-1}\left(P_{i j}^{k l} \partial_{k} \partial_{l} L+Q_{i j}^{k l} g_{k l}\right) \partial_{i} h \partial_{j} h w^{p} \psi^{2} d g
$$


After integration by parts, this becomes

$$
\begin{array}{rl}
I_{3}=10 & n p(5 n p-1) \int F^{5 n p-2} \partial_{k} F P_{i j}^{k l} \partial_{l} L \partial_{i} h \partial_{j} h w^{p} \psi^{2} d g \\
& +10 n p \int F^{5 n p-1} P_{i j}^{k l} \partial_{l} L\left(\partial_{k} \partial_{i} h\right) \partial_{j} h w^{p} \psi^{2} d g \\
& +10 n p \int F^{5 n p-1} P_{i j}^{k l} \partial_{l} L \partial_{i} h\left(\partial_{k} \partial_{j} h\right) w^{p} \psi^{2} d g \\
& +10 n p \int F^{5 n p-1} P_{i j}^{k l} \partial_{l} L \partial_{i} h \partial_{j} h \partial_{k}\left(w^{p} \psi\right) \psi d g \\
& +10 n p \int F^{5 n p-1} P_{i j}^{k l} \partial_{l} L \partial_{i} h \partial_{j} h w^{p} \psi \partial_{k} \psi \\
& \quad-10 n p \int F^{5 n p-1} Q_{i j}^{k l} g_{k l} \partial_{i} h \partial_{j} h w^{p} \psi^{2} d g \\
\equiv T_{1} & +\ldots+T_{6} .
\end{array}
$$

Let us bound $T_{i}, i=1, \ldots, 6$. Observe that

$$
\left|T_{1}\right| \leq 10 n p(5 n p-1)\|\nabla L\|_{\infty} \int F^{5 n p-2}|\nabla F||\nabla h|^{2} w^{p} \psi^{2} d g .
$$

Since $|\nabla h|^{2}=F$, we deduce, using $w^{p}=F^{5 n p}$,

$$
\begin{aligned}
\left|T_{1}\right| & \leq 10 n p(5 n p-1)\|\nabla L\|_{\infty} \int F^{5 n p-1}|\nabla F| w^{p} \psi^{2} d g \\
& \leq 10 n p\|\nabla L\|_{\infty} \int\left|\nabla w^{p}\right| w^{p} \psi^{2} d g .
\end{aligned}
$$

Thus, after a little calculation, we obtain,

$$
\left|T_{1}\right| \leq \frac{1}{10} \int\left|\nabla\left(w^{p} \psi\right)\right|^{2} d g+c p^{2}\|\nabla L\|_{\infty}^{2} \int w^{2 p} \psi^{2} d g+c\|\nabla \psi\|_{\infty}^{2} \int_{\text {supp } \psi} w^{2 p} d g .
$$

Next

$$
\begin{aligned}
\left|T_{2}\right| & \leq 10 n p\|\nabla L\|_{\infty} \int F^{5 n p-1} \mid \text { Hess } h|| \nabla h \mid w^{p} \psi^{2} d g \\
& \leq n p \int F^{5 n p-1} \mid \text { Hess }\left.h\right|^{2} w^{p} \psi^{2} d g+c n p\|\nabla L\|_{\infty}^{2} \int F^{5 n p-1}|\nabla h|^{2} w^{p} \psi^{2} d g .
\end{aligned}
$$

Recalling again that $|\nabla h|^{2}=F$ and the definition of $I_{1}$, we deduce

$$
\left|T_{2}\right| \leq-\frac{I_{1}}{10}+c n p\|\nabla L\|_{\infty}^{2} \int w^{2 p} \psi^{2} d g .
$$

Since $T_{3}$ is similar to $T_{2}$, we also have

$$
\left|T_{3}\right| \leq-\frac{I_{1}}{10}+c n p\|\nabla L\|_{\infty}^{2} \int w^{2 p} \psi^{2} d g .
$$

By Young's inequality

$$
\left|T_{4}\right| \leq \frac{1}{2} \int\left|\nabla\left(w^{p} \psi\right)\right|^{2} d g+50 n^{2} p^{2}\|\nabla L\|_{\infty}^{2} \int F^{10 n p-2}|\nabla h|^{4} \psi^{2} d g .
$$


Since $F=|\nabla h|^{2}$ and $w=F^{5 n}$, this shows

$$
\left|T_{4}\right| \leq \frac{1}{2} \int\left|\nabla\left(w^{p} \psi\right)\right|^{2} d g+c p^{2}\|\nabla L\|_{\infty}^{2} \int w^{2 p} \psi^{2} d g .
$$

Next

$$
\left|T_{5}\right| \leq 10 n p\|\nabla L\|_{\infty}\|\psi\|_{\infty} \int F^{5 n p-1}|\nabla h|^{2} w^{p} \psi d g
$$

which becomes

$$
\left|T_{5}\right| \leq 10 n p\|\nabla L\|_{\infty}\|\psi\|_{\infty} \int w^{2 p} \psi d g
$$

Lastly

$$
\left|T_{6}\right| \leq 10 n p \int F^{5 n p-1}|\nabla h|^{2} w^{p} \psi^{2} d g=10 n p \int w^{2 p} \psi^{2} d g .
$$

Substituting (2.16)-(2.21) into (2.15), we find that

$$
\left|I_{3}\right| \leq \frac{\left|I_{1}\right|}{5}+\frac{3}{5} \int\left|\nabla\left(w^{p} \psi\right)\right|^{2} d g+c \frac{p^{2}\|\nabla L\|_{\infty}^{2}+1}{[(1-\sigma) r]^{2}} \int_{\text {supp } \psi} w^{2 p} d g .
$$

Here we recall that

$$
I_{1}=-10 n p \int F^{5 n p-1}|H e s s h|^{2} w^{p} \psi^{2} d g
$$

Using the inequality

$$
\mid H \text { ess }\left.h\right|^{2} \geq \frac{1}{n}(\Delta h)^{2}=\frac{1}{n}|\nabla h|^{4},
$$

we find that

$$
I_{1}=\frac{I_{1}}{2}+\frac{I_{1}}{2} \leq \frac{I_{1}}{2}-5 p \int F^{5 n p-1}|\nabla h|^{4} w^{p} \psi^{2} d g
$$

which induces, since $w=F^{5 n}$ and $F=|\nabla h|^{2}$, that

$$
I_{1} \leq \frac{I_{1}}{2}-5 p \int F w^{2 p} \psi^{2} d g .
$$

Substituting (2.23), (2.22) and (2.14) into (2.13), we deduce

$$
\begin{gathered}
\int\left|\nabla\left(w^{p} \psi\right)\right|^{2} d g \leq \frac{I_{1}}{2}-5 p \int F w^{2 p} \psi^{2} d g+(\epsilon+1) \int F w^{2 p} \psi^{2} d g+\epsilon^{-1}\|\nabla \psi\|_{\infty}^{2} \int w^{2 p} \psi^{2} d g \\
+\frac{\left|I_{1}\right|}{5}+\frac{3}{5} \int\left|\nabla\left(w^{p} \psi\right)\right|^{2} d g+c \frac{p^{2}\|\nabla L\|_{\infty}^{2}+1}{[(1-\sigma) r]^{2}} \int_{\text {supp } \psi} w^{2 p} d g .
\end{gathered}
$$

Since $p \geq 1$, we can take $\epsilon=1$ and obtain

$$
\int\left|\nabla\left(w^{p} \psi\right)\right|^{2} d g+\int\left(w^{p} \psi\right)^{2} d g \leq c \frac{p^{2}\|\nabla L\|_{\infty}^{2}+1}{[(1-\sigma) r]^{2}} \int_{\text {supp } \psi} w^{2 p} d g
$$

where $c$ may have changed in value. By the Sobolev inequality in Assumption 1, this implies

$$
\left(\int\left(w^{p} \psi\right)^{2 n /(n-2)} d g\right)^{(n-2) / n} \leq c \alpha \frac{p^{2}\|\nabla L\|_{\infty}^{2}+1}{[(1-\sigma) r]^{2}} \int_{\text {supp } \psi} w^{2 p} d g .
$$


From this, the standard Moser's iteration implies

$$
\sup _{B(x, \sigma r)} w^{2} \leq \frac{C\left(\alpha, n,\|\nabla L\|_{\infty}^{2}\right)}{(1-\sigma)^{n} r^{n}} \int_{B(x, r)} w^{2} d g
$$

for $r, \sigma \in(0,1]$. Using $w=F^{5 n}$, we arrive at

$$
\sup _{B(x, \sigma r)} F \leq\left(\frac{C\left(\alpha, n,\|\nabla L\|_{\infty}^{2}\right)}{(1-\sigma)^{n} r^{n}} \int_{B(x, r)} F^{10 n} d g\right)^{1 /(10 n)}
$$

for $r, \sigma \in(0,1]$. Using the volume doubling property and an algebraic trick in [LS] e.g., we deduce

$$
\sup _{B(x, r / 2)} F \leq \frac{C\left(\alpha, n,\|\nabla L\|_{\infty}^{2}\right)}{r^{n}} \int_{B(x, r)} F d g
$$

for $r, \sigma \in(0,1]$. Using integration by parts, it is known that

$$
\int_{B(x, r)} F d g=\int_{B(x, r)}|\nabla(\ln u)|^{2} d g \leq 4 \frac{|B(x, 4 r)|}{r^{2}} \leq c r^{n-2}
$$

where we have used Assumption 2. Substituting this to (2.24), we arrive at

$$
\sup _{B(x, r / 2)}|\nabla(\ln u)| \leq \frac{C\left(\alpha, n,\|\nabla L\|_{\infty}^{2}\right)}{r}
$$

proving the proposition.

\section{Proof of the Theorem}

Proof. (Theorem 1.1).

For simplicity of presentation, we omit the time variable in the proof. It is also clear that we can take $\bar{u}=0$.

Step 1.

Pick $u \in C^{\infty}(\mathbf{M})$. Since $\Delta u=\Delta u$ and $\bar{u}=0$, we have

$$
u(x)=-\int_{\mathbf{M}} \Gamma_{0}(x, y) \Delta u(y) d g(y)
$$

where $\Gamma_{0}$ is the Green's function of the Laplacian on M. Pick a small balls $B(x, r)$. Then,

$$
\begin{aligned}
u(x) & =-\lim _{r \rightarrow 0} \int_{\mathbf{M}-B(x, r)} \Gamma_{0}(x, y) \Delta u(y) d g(y) \\
& =\lim _{r \rightarrow 0} \int_{\mathbf{M}-B(x, r)} \nabla \Gamma_{0}(x, y) \nabla u(y) d g(y)-\lim _{r \rightarrow 0} \int_{\partial B(x, r)} \Gamma_{0}(x, y) \partial_{n} u(y) d S .
\end{aligned}
$$

Here we have used integration by parts. Note that $\left|\Gamma_{0}(x, y)\right| \leq \frac{C_{0}}{d(x, y)^{n-2}}$ by Lemma2.3. Also the volume of $\partial B(x, r)$, the small spheres of radius $r$, is bounded from above by $\mathrm{Cr}^{n-1}$. So the second limit is 0 . We mention that one does not need a uniform in time bound for $|\partial B(x, r)|$ since we are freezing a time and taking the limit $r \rightarrow 0$. Hence

$$
u(x)=\int_{\mathbf{M}} \nabla \Gamma_{0}(x, y) \nabla u(y) d g(y) .
$$


According to Lemma 2.3, this implies

$$
|u(x)| \leq C_{0} \int_{\mathbf{M}} \frac{|\nabla u(y)|}{d(x, y)^{n-1}} d g(y) \equiv C_{0} I_{1}(|\nabla u|)(x) .
$$

Here $I_{1}$ is the Riesz potential of order 1 .

We claim that there exists a constant $C_{1}$, depending only on the constant $\kappa$ in Property A 5, such that

$$
\left|I_{1}(f)(x)\right| \leq C_{1}[M(f)(x)]^{1-(1 / n)}\|f\|_{1}^{1 / n} .
$$

for all smooth function $f$ on $\mathbf{M}$. Here $M(f)$ is the Hardy-Littlewood maximal function. The proof given here is more or less the same as in the Euclidean case (p86 [Zi]), under Property A 5, i.e. $\kappa r^{n} \leq|B(x, r)| \leq \kappa^{-1} r^{n}$. Let $\delta$ be a positive number, then

$$
\begin{aligned}
\left|I_{1}(f)(x)\right| & \leq \int_{B(x, \delta)} \frac{|f(y)|}{d(x, y)^{n-1}} d g+\int_{B^{c}(x, \delta)} \frac{|f(y)|}{d(x, y)^{n-1}} d g \\
& \leq \Sigma_{j=0}^{\infty} \int_{\left\{2^{-j-1} \delta \leq d(x, y)<2^{-j} \delta\right\}} \frac{|f(y)|}{d(x, y)^{n-1}} d g+\delta^{1-n} \int_{\mathbf{M}}|f(y)| d g \\
& \leq \Sigma_{j=0}^{\infty}\left(2^{(j+1)} / \delta\right)^{n-1}\left|B\left(x, 2^{-j} \delta\right)\right| \frac{1}{\left|B\left(x, 2^{-j} \delta\right)\right|} \int_{B\left(x, 2^{-j} \delta\right)}|f(y)| d g+\delta^{1-n} \int_{\mathbf{M}}|f(y)| d g \\
& \leq \Sigma_{j=0}^{\infty}\left(2^{(j+1)} / \delta\right)^{n-1}\left|B\left(x, 2^{-j} \delta\right)\right| M(f)(x)+\delta^{1-n}\|f\|_{1} .
\end{aligned}
$$

By Property A 5,

$$
\left|B\left(x, 2^{-j} \delta\right)\right| \leq \kappa^{-1}\left(2^{-j} \delta\right)^{n}
$$

Combining the last 2 inequalities we deduce

$$
\left|I_{1}(f)(x)\right| \leq C \kappa^{-1} \delta M(f)(x)+\delta^{1-n}\|f\|_{1}
$$

which implies (3.2) by taking $\delta=\left[M(f)(x) /\|f\|_{1}\right]^{-1 / n}$. We remark that if $\delta>\operatorname{diam}(\mathbf{M})$, then the integral $\int_{B^{c}(x, \delta)} \frac{|f(y)|}{d(x, y)^{n-1}} d g$ is regarded as zero.

Since Property A 4-5 induces volume doubling property, it is well known that the maximal operator is bounded from $L^{1}(\mathbf{M})$ to weak $L^{1}(\mathbf{M})$, i.e. there is a positive constant $C_{2}$, depending only on $\kappa$ such that

$$
\beta|\{x \mid M(f)(x)>\beta\}| \leq C_{2}\|f\|_{1},
$$

for all $\beta>0$. A short proof can be found in Chapter 3 of Folland's book [Fo] e.g. Note the proof there is written for the Euclidean space. But as indicated below, it is clear that it works for all metric spaces with volume doubling property. Pick $x \in S_{\beta} \equiv\{x \mid M(f)(x)>$ $\beta$. Then by definition of $M(f)(x)$, there exists radius $r_{x}>0$ such that

$$
\frac{1}{\left|B\left(x, r_{x}\right)\right|} \int_{B\left(x, r_{x}\right)}|f(y)| d g>\beta .
$$

Note that the family of balls $\left\{B\left(x, r_{x}\right) \mid x \in S_{\beta}\right\}$ is an open cover of $S_{\beta}$. Since the manifold is compact, by well known covering argument for compact metric spaces, there exists a finite subfamily $\left\{B\left(x, r_{x_{i}}\right) \mid i=1, \ldots, m\right\}$ of disjoint balls such that $\left\{B\left(x, 3 r_{x_{i}}\right) \mid i=1, \ldots, m\right\}$ covers $S_{\beta}$. Using volume doubling property, one has

$$
\beta\left|S_{\beta}\right| \leq \beta \Sigma_{i}\left|B\left(x, 3 r_{x_{i}}\right)\right| \leq C \Sigma_{i} \beta\left|B\left(x, r_{x_{i}}\right)\right| \leq\|f\|_{1} .
$$


Combining this with (3.2), we obtain, for all $\alpha>0$,

$$
\begin{aligned}
\left|\left\{x \mid I_{1}(f)(x)>\alpha\right\}\right| & \leq\left|\left\{x \mid M(f)(x)>\frac{\alpha^{n /(n-1)}}{\|f\|_{1}^{1 /(n-1)} C_{1}^{n /(n-1)}}\right\}\right| \\
& \leq C_{2} C_{1}^{n /(n-1)}\|f\|_{1}^{1 /(n-1)} \alpha^{-n /(n-1)}\|f\|_{1} .
\end{aligned}
$$

Thus

$$
\alpha^{n /(n-1)}\left|\left\{x \mid I_{1}(f)(x)>\alpha\right\}\right| \leq C_{2} C_{1}^{n /(n-1)}\|f\|_{1}^{n /(n-1)}
$$

By (3.1) we have

$$
|\{x|| u(x) \mid>\alpha\} \leq|\left\{x|| I_{1}(\nabla u)(x) \mid>\alpha C_{0}^{-1}\right\},
$$

which infers, via (3.3) with $f=|\nabla u|$ the following statement:

if $\bar{u}=0$ then for all $\alpha>0$, it holds

$$
\alpha^{n /(n-1)}|\{x|| u(x) \mid>\alpha\}| \leq C_{3}\|\nabla u\|_{1}^{n /(n-1)} .
$$

Here $C_{3}$ is a constant depending only on the controlling constants in Properties A and B. Step 2.

Now we will convert the weak type inequality (3.4) to the desired $L^{1}$ Sobolev inequality, using an argument based on the idea in [FGW]. See also [CDG]. Define the sets

$$
D_{k}=\left\{x|| u(x) \mid>2^{k}\right\}, \quad k \text { are integers. }
$$

Then

$$
\|u\|_{p}=\left(\Sigma_{k=-\infty}^{\infty} \int_{D_{k}-D_{k+1}}|u(x)|^{p} d g\right)^{1 / p}
$$

where $p=n /(n-1)$ here and later in the proof. This shows

$$
\|u\|_{p} \leq\left(\Sigma_{k=-\infty}^{\infty} 2^{(k+1) p}\left|D_{k}\right|\right)^{1 / p}=\left(\Sigma_{k=-\infty}^{\infty} 2^{(k+1) p}\left|\left\{x|| u(x) \mid>2^{k}\right\}\right|\right)^{1 / p} .
$$

Now we define

$$
g_{k}=g_{k}(x)=\left\{\begin{array}{l}
2^{k-1}, \quad x \in D_{k}=\left\{x|| u(x) \mid>2^{k}\right\}, \\
|u(x)|-2^{k-1}, \quad x \in D_{k-1}-D_{k}=\left\{x\left|2^{k-1}<\right| u(x) \mid \leq 2^{k}\right\}, \\
0, \quad x \in D_{k-1}^{c}=\left\{x|| u(x) \mid \leq 2^{k-1}\right\} .
\end{array}\right.
$$

It is clear that $g_{k}$ is a Lipschitz function such that $0 \leq g_{k} \leq|u| / 2$.

Observe that

$D_{k} \subset\left\{x \mid g_{k}(x)=2^{k-1}\right\} \subset\left\{x \mid g_{k}(x)>2^{k-2}\right\} \subset\left\{x|| g_{k}(x)-\bar{g}_{k} \mid>2^{k-3}\right\} \cup\left\{x \mid \bar{g}_{k}>2^{k-3}\right\}$

Here $\bar{g}_{k}$ is the average of $g_{k}$ on $\mathbf{M}$. Hence

$$
\begin{aligned}
\left|D_{k}\right| & \leq\left|\left\{x|| g_{k}(x)-\bar{g}_{k} \mid>2^{k-3}\right\}\right|+\left|\left\{x \mid \bar{g}_{k}>2^{k-3}\right\}\right| \\
& \equiv T_{k 1}+T_{k 2} .
\end{aligned}
$$

Note the average of the function $g_{k}-\bar{g}_{k}$ is 0 . Thus we can apply (3.4), with $u$ there being replaced by $g_{k}-\bar{g}_{k}$, to deduce

$$
T_{k 1}=\left|\left\{x|| g_{k}(x)-\bar{g}_{k} \mid>2^{k-3}\right\}\right| \leq C C_{3} 2^{-p k}\left\|\nabla g_{k}\right\|_{1}^{p} .
$$


To treat $T_{k 2}$, recall that $g_{k} \leq|u| / 2$ which implies

$$
\bar{g}_{k} \leq\|u\|_{1} /(2|\mathbf{M}|) \text {. }
$$

Therefore

This shows that

$$
T_{k 2}=\left|\left\{x \mid \bar{g}_{k}>2^{k-3}\right\}\right| \leq\left|\left\{x \mid \frac{\|u\|_{1}}{|\mathbf{M}|}>2^{k-2}\right\}\right|
$$

$$
T_{k 2}=\left\{\begin{array}{l}
0, \quad \text { when } \quad k>2+\log _{2} \frac{\|u\|_{1}}{|\mathbf{M}|} \equiv k_{0} \\
|\mathbf{M}|, \quad k \leq k_{0} .
\end{array}\right.
$$

Substituting (3.7) and (3.8) into (3.6), we deduce

$$
\left|D_{k}\right| \leq\left\{\begin{array}{l}
C C_{3} 2^{-p k}\left\|\nabla g_{k}\right\|_{1}^{p}, \quad \text { when } \quad k>k_{0} \\
C C_{3} 2^{-p k}\left\|\nabla g_{k}\right\|_{1}^{p}+|\mathbf{M}|, \quad k \leq k_{0} .
\end{array}\right.
$$

Substituting this to (3.5) and using Minkowski inequality, we obtain

$$
\|u\|_{p} \leq C_{4} \Sigma_{k=-\infty}^{\infty}\left\|\nabla g_{k}\right\|_{1}+C|\mathbf{M}|^{1 / p} \Sigma_{k=-\infty}^{\left[k_{0}\right]+1} 2^{k}
$$

Here $\left[k_{0}\right]$ is the greatest integer less than or equal to $k_{0}$. Note that the supports of $\nabla g_{k}$ are disjoint and $\nabla g_{k}=\nabla|u|$ in the supports. Also by the definition of $k_{0}$ in (3.8), we have $2^{k_{0}}=4\|u\|_{1} /|\mathbf{M}|$. Hence

$$
\|u\|_{p} \leq C_{4}\|\nabla u\|_{1}+C|\mathbf{M}|^{1 / p}\|u\|_{1} /|\mathbf{M}|,
$$

which implies, since $p=n /(n-1)$,

$$
\|u\|_{n /(n-1)} \leq C_{4}\|\nabla u\|_{1}+C \frac{1}{|\mathbf{M}|^{1 / n}}\|u\|_{1} .
$$

Here $C$ is a numerical constant. This proves Theorem 1.1.

Two final remarks are in order. Let $\alpha$ be the average of $u$ in $\mathbf{M}$. Then By Theorem 1.1, we have

$$
\|u-\alpha\|_{n /(n-1)} \leq C\|\nabla u\|_{1}+C \frac{1}{|\mathbf{M}|^{1 / n}}\|u-\alpha\|_{1} .
$$

Since the average of $u-\alpha$ is zero, inequality (3.1) implies

$$
|u(x)-\alpha| \leq C_{0} \int_{\mathbf{M}} \frac{|\nabla u(y)|}{d(x, y)^{n-1}} d g(y) .
$$

After integration, using the $\kappa$ noninflating property, we find that

$$
\|u-\alpha\|_{1} \leq \operatorname{Cdiam}(\mathbf{M})\|\nabla u\|_{1} .
$$

By the $\kappa$ noncollapsing property of $\mathbf{M}$, there holds $\operatorname{diam}(\mathbf{M}) \leq C|\mathbf{M}|^{1 / n}$. This shows the usual isoperimetric inequality

$$
\|u-\alpha\|_{n /(n-1)} \leq C\|\nabla u\|_{1} .
$$

Notice the $L^{2}$ Poincaré inequality (2.7), [Z11] and Section 9 of [Ch] imply the following long time convergence result: the Kähler Ricci flow in Theorem 1.1 converges sequentially in time, under Gromov-Hausdorff topology, to a compact metric space with $L^{2}$ Poincaré inequality and volume doubling condition. By Cheeger's Theorem 11.7 [Ch], the limit space can be equipped with differential structure a.e.. 
Acknowledgment. Q. S. Z. would like to thank Professors L. Capogna, X. X. Chen, D. Jerison, Bing Wang and Bun Wong for helpful conversations. Part of the work was done when he was a visiting professor at Nanjing University under a Siyuan Foundation grant, the support of which is gratefully acknowledged.

Both of us wish to thank the referee for checking the paper carefully and making helpful corrections and suggestions.

\section{REFERENCES}

[Ca] Cao, H. D. Deformation of Kähler metrics to Kähler-Einstein metrics on compact Kähler manifolds, Invent. Math. 81 (1985), 359-372.

[CCZ] Cao, H.D., Chen, B. L. and Zhu, X. P., Ricci flow on compact Kähler manifold of positive bisectional curvatute, math.DG/0302087 C.R.A.S. 337:12 (2003), 781-784.

[Ch] Cheeger, J., Differentiability of Lipschitz functions on metric measure spaces, GAFA, Vol. 9 (1999) 428-517.

[CD] Coulhon, Thierry; Duong, Xuan, Thinh Riesz transforms for $1 \leq p \leq 2$. Trans. Amer. Math. Soc. 351 (1999), no. 3, 1151-1169.

[CDG] Capogna, Luca; Danielli, Donatella; Garofalo, Nicola, The geometric Sobolev embedding for vector fields and the isoperimetric inequality. Comm. Anal. Geom. 2 (1994), no. 2, 203-215.

[CLN] Bennett Chow, Peng Lu and Lei Ni, Hamilton's Ricci flow, Science Press Beijing/AMS, 2006.

[CT1] Xiuxiong Chen, Gang Tian, Ricci flows on Kähler Einstein surfaces, Invent. Math., 147 (2002) $487-544$.

[CT2] Xiuxiong Chen, Gang Tian, Ricci flows on Kähler Einstein manifolds, Duke Math. J., 131 (2006) no. $1,17-73$.

[CW] Xiuxiong Chen, Bing Wang, Space of Ricci flows (I), arXiv:0902.1545, Comm. Pure Appl. Math. 65 (2012), no. 10, 1399-1457.

[CW2] Xiuxiong Chen, Bing Wang On the conditions to extend Ricci flow(III), arXiv:1107.5110

[CY] Cheng, S. Y.; Yau, S. T. Differential equations on Riemannian manifolds and their geometric applications. Comm. Pure Appl. Math. 28 (1975), no. 3, 333-354.

[J] Jerison, David, The Poincaré inequality for vector fields satisfying Hörmander's condition. Duke Math. J. 53 (1986), no. 2, 503-523.

[FGW] Franchi, B.; Gallot, S.; Wheeden, R. L. Sobolev and isoperimetric inequalities for degenerate metrics. Math. Ann. 300 (1994), no. 4, 557-571.

[Fo] G. Folland, Real Analysis, Wiley, 1999.

[Gr] Grigorýan, A. A. The heat equation on noncompact Riemannian manifolds. (Russian) Mat. Sb. 182 (1991), no. 1, 55-87; translation in Math. USSR-Sb. 72 (1992), no. 1, 47-77.

[Ha] Hamilton, R. S. An isoperimetric estimate for Ricci flow on the two sphere, Ann. of Math. Stud., 137, p191-200, Princeton Univ. Press, 1995.

[LS] Li, Peter; Schoen, Richard, $L^{p}$ and mean value properties of subharmonic functions on Riemannian manifolds. Acta Math. 153 (1984), no. 3-4, 279-301.

[P] Perelman, Grisha, The entropy formula for the Ricci flow and its geometric applications, Math. ArXiv, math.DG/0211159.

[Sa] Saloff-Coste, L. A note on Poincaré, Sobolev, and Harnack inequalities. Internat. Math. Res. Notices 1992, no. 2, 27-38.

[Se] Sesum, Natasa, Convergence of a Kähler-Ricci flow. Math. Res. Lett. 12 (2005), no. 5-6, 623-632.

[ST] Sesum, Natasa; Tian, Gang, Bounding scalar curvature and diameter along the Kähler Ricci flow (after Perelman). J. Inst. Math. Jussieu 7 (2008), no. 3, 575-587.

[Ye] Rugang Ye, The logarithmic Sobolev inequality along the Ricci flow, arXiv:0707.2424.

[Z07] Qi S. Zhang, A uniform Sobolev inequality under Ricci flow, IMRN 2007, ibidi Erratum, Addendum.

[Z10] Qi S. Zhang. Sobolev inequalities, heat kernels under Ricci flow and the Poincaré conjecture. CRC Press, Boca Raton, FL, 2011.

[Z11] Qi S. Zhang. Bounds on volume growth of geodesic balls under Ricci flow, arXiv:1107.4262, Math. Res. Lett., Vol. 19: 1 p.245-253, (2012). 
[Zi] Ziemer, William P. Weakly differentiable functions. Sobolev spaces and functions of bounded variation. Graduate Texts in Mathematics, 120. Springer-Verlag, New York, 1989. xvi+308 pp.

e-mail: tian@math.princeton.edu and qizhang@math.ucr.edu

BiCmR, Peking University, Beijing, 100871, China and Department of Mathematics, Princeton University, Princeton, NJ 02139, USA

Department of Mathematics Nanjing University, Nanjing 210093, China; Department of Mathematics, University of California, Riverside, CA 92521, USA 